\title{
Left Ventricular Events in Systemic Hypertension
}

\author{
V. PARAMESHVARA AND S. P. SINGH \\ From the General Hospital, and The United Birmingham Hospitals, and Birmingham University
}

The duration of the various mechanical events of ventricular activity is considerably modified by such factors as heart rate, stroke volume, sympathomimetic agents, and body temperature. The time relationship of these events with the variations of the aortic pressure curve has been determined by several workers (Weissler, Peeler, and Roehll, 1961 ; Braunwald, Sarnoff, and Stainsby, 1958; Shah and Slodki, 1964), with inconsistent results. It is not possible, even in isolated hearts, to change a single hæmodynamic variable without altering others, which makes it difficult to study the effect of each factor separately (Wallace et al., 1963).

The present work deals with the influence of raised arterial pressure on the left ventricular events in hypertensive subjects.

\section{SUBJECTS AND METHOD}

Studies were made on 20 patients with essential hypertension between 27 and 67 years. There were 15 men and 5 women. The diastolic blood pressure ranged from 110 to $155 \mathrm{~mm}$. Hg. The patients were classified according to Keith, Wagener, and Barker's (1939) criteria as grade III or IV and had been on hypotensive therapy for varying periods. All were ambulatory and denied exertional intolerance. Left ventricular hypertrophy was confirmed in all cases by chest radiograph and electrocardiogram; and the serum electrolytes were normal.

Simultaneous tracings were obtained of electrocardiogram, carotid artery pulse, and phonocardiograms from two places (apex and second right interspace parasternally), with the patients supine and at the end of expiration. The experiment was repeated after mild exercise, which consisted of raising the legs against gravity. This was sufficient to decrease the mean $R-R$ interval by 115.9 milliseconds.

A six-channel NEP photographic recorder was used with a paper speed of $80 \mathrm{~mm}$. per second and at a high frequency filter ( 200 cycles per second).

Received January 20, 1966.
The following measurements were recorded at rest and after exercise: $R-R$ interval; $Q-M_{1}$ time (onset of QRS to mitral component of first heart sound); period of isometric contraction (mitral component of first heart sound to the beginning of upstroke in carotid pulse); the upstroke time and $Q-A_{2}$ interval (onset of $Q R S$ to aortic component of second heart sound).

\section{Results}

The relation between various systolic left ventricular events recorded at the end of the expiratory phase, both at rest and after exercise, has been explored by regression analysis.

We have selected three sets for discussion here. Larger numbers of observations might have justified a fuller discussion of such interrelationships.

Regression lines have been fitted to the data shown in Fig. 1, 2, and 3.

(i) $Q-A_{2}$ and square root of $R-R$ (Fig. 1). At rest: $\mathrm{Q}-\mathrm{A}_{2}=109.5+10.0 \sqrt{\mathrm{R}-\mathrm{R}}$ (correlation coefficient $=0.47 ; \mathrm{p}<0.05$ ).

After exercise: $\mathrm{Q}-\mathrm{A}_{2}=148 \cdot 1+8.0 \sqrt{\mathrm{R}-\mathrm{R}}$ (correlation coefficient $=0.52 ; \mathrm{p}<0.05$ ).

(ii) Ejection period (EP) and $R-R$ (Fig. 2).

At rest: $\mathrm{EP}=156+0 \cdot 15 \mathrm{RR}$ (correlation coefficient $=0.60 ; \mathrm{p}<0.01$ ).

After exercise: $\mathrm{EP}=179+0 \cdot 11 \mathrm{RR}$ (correlation coefficient $=0.53 ; \mathrm{p}<0.05)$.

(iii) Isometric contraction (IP) and $\left(Q-A_{2}\right)$ (Fig. 3).

At rest: $\mathrm{IP}=0.20\left(\mathrm{Q}-\mathrm{A}_{2}\right)-25.44$ (correlation coefficient $=0.48 ; \mathrm{p}<0.05)$.

After exercise: IP $=0 \cdot 14\left(\mathrm{Q}-\mathrm{A}_{2}\right)-5 \cdot 12$ (correlation coefficient $=0.46 ; p<0.05)$.

We could find no evidence in these data of a 

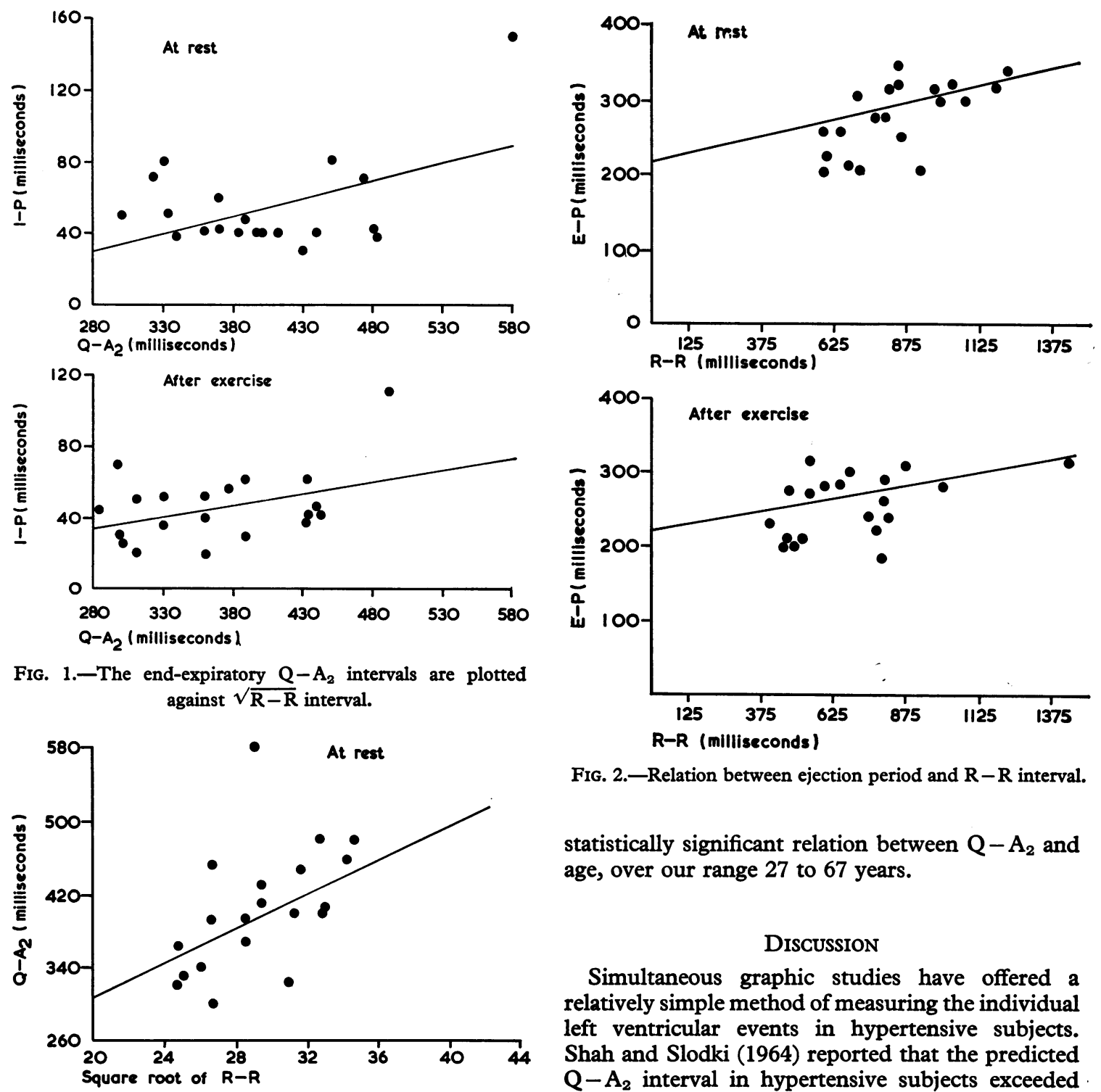

Fig. 2.-Relation between ejection period and $\mathbf{R}-\mathbf{R}$ interval.

statistically significant relation between $Q-A_{2}$ and age, over our range 27 to 67 years.

\section{Discussion}

Simultaneous graphic studies have offered a relatively simple method of measuring the individual left ventricular events in hypertensive subjects. Shah and Slodki (1964) reported that the predicted $\mathbf{Q}-\mathbf{A}_{\mathbf{2}}$ interval in hypertensive subjects exceeded that of normal persons by 33.4 milliseconds. In

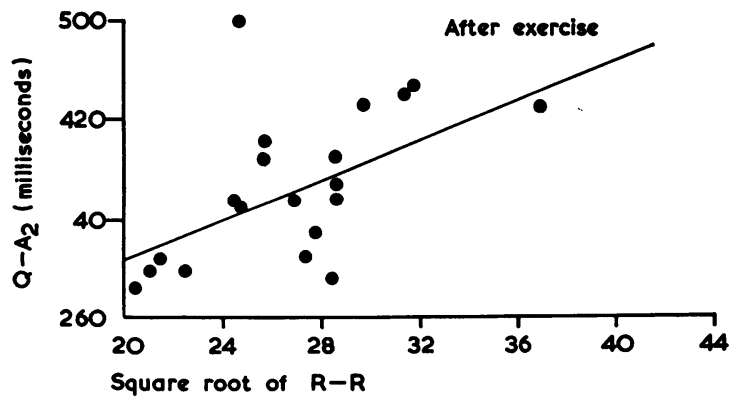

FIG. 3.-Relation between isometric contraction period and $\mathbf{R}-\mathbf{R}$ interval. value of one standard deviation for the predicted $Q-A_{2}$ was $17 \cdot 5$. Our results are in close accord with these findings. After exercise a linear dependence of $Q-A_{2}$ on the square root of $R-R$ was maintained, though the mean $Q-A_{2}$ period was reduced by 37.4 milliseconds. The influence of raised aortic pressure on ventricular systole is not exactly known. Wallace et al. (1963) found that if heart rate and stroke volume were kept constant a sudden rise in mean aortic pressure had no measurable effect on the duration of the phases of systole. They also noted that a decreased duration of ejection 
was not necessarily associated with a raised ventricular end-diastolic pressure.

Our experience of hypertensive patients indicates a different kind of response. Comparing our data with those of normal persons studied by Shah and Slodki (1964), the predicted $Q-A_{2}$ interval is greater by 47 milliseconds in hypertensive patients. This prolongation of left ventricular systole might be due to the myocardial contraction against increased peripheral resistance.

Animal experiments demonstrated that pronounced increase of resistance to ventricular ejection resulted in its prolongation (Braunwald et al., 1958). Weissler et al. (1961) observed a reduction in ejection time relative to stroke volume in uncomplicated hypertensive patients. In dogs an increase of aortic pressure shortened the ejection time and prolonged the isometric contraction period. Leonard, Weissler, and Warren (1957) studied the period of isometric contraction in 25 hypertensive patients and found it to be prolonged (mean value $0.07 \mathrm{sec}$. S.D. $= \pm 0.01$ ). They explained this prolongation on the hypothesis that higher aortic pressure had to be overcome before the ventricle ejected the blood across the aortic valves. In the present study the mean value for isometric contraction period is 54 milliseconds at rest and 45 milliseconds after exercise. These values are within the normal range. Presumably a hypertrophied ventricle takes little, if any, more time in overcoming the raised aortic pressure. Prolongation of $Q-A_{2}$ interval is perhaps due, in part, to the slower passage of the electrical impulse through the hypertrophied ventricle.

Benchimol, Dimond, and Shen (1960) estimated the total ejection time between 280 to 340 milliseconds in 30 normal subjects (mean $=302 \pm 0.017$ ). Evidence in the present experiment indicates that the main delay in systole occurs during the ejection phase ( 300 milliseconds in 14 patients and 280 or less in 6 patients). Mean upstroke time for normal persons is calculated to be $88 \pm 13$ milliseconds (Benchimol et al., 1960). In the present group the mean upstroke time is $185 \cdot 1 \pm 12.05$ milliseconds at rest and $154 \cdot 1 \pm 15 \cdot 4$ milliseconds after exercise. These values are more than doubled in our hypertensives compared to the normal subjects. A constant stroke output can be maintained only by attaining a sufficient gradient across the aortic valves. If other variants are constant, the duration of upstroke time and consequently that of systole should be directly proportional to the degree of systemic hypertension.

\section{SUMMARY}

Left ventricular events were studied by using simultaneous graphic records in 20 hypertensive patients. The Q- $\mathrm{A}_{2}$ interval was found to be prolonged, and the delay was most significant during upstroke time of the ejection phase.

We are grateful to Dr. Clifford Parsons for his encouragement and criticism. We should like to acknowledge the help with the statistical analysis which we received from Mrs Susan Farrington and Dr. J. A. H. Waterhouse of the Department of Social Medicine, Birmingham University.

\section{REFERENCES}

Benchimol, A., Dimond, E. G., and Shen, Y. (1960). Ejection time in aortic stenosis and mitral stenosis. Amer. $\mathcal{F}$. Cardiol., 5, 728.

Braunwald, E., Sarnoff, S. J., and Stainsby, W. N. (1958). Determinants of duration and mean rate of ventricular ejection. Circulat. Res., 6, 319.

Keith, N. M., Wagener, H. P., and Barker, N. W. (1939). Some different types of essential hypertension: Their course and prognosis. Amer. F. med. Sci., 197, 332.

Leonard, J. J., Weissler, A. M., and Warren, J. V. (1957). Observations on the significance of the delayed appearance of the first heart sound in mitral stenosis. Circulation, 16, 906.

Shah, P. M., and Slodki, S. J. (1964). The Q-II interval. A study of the second heart sound in normal adults and in systemic hypertension. Circulation, 29, 551.

Wallace, A. G., Mitchell, J. H., Skinner, N. S., and Sarnoff, S. J. (1963). Duration of the phases of left ventricular systole. Circulat. Res., 12, 611.

Weissler, A. M., Peeler, R. G., and Roehll, W. H., Jr. (1961). Relationship between left ventricular ejection time, stroke volume, and heart rate in normal individuals and in patients with cardiovascular disease. Amer. Heart f., 62, 367. 